\title{
Imaging and cytological analysis of 92 patients with Japanese encephalitis
}

\author{
Qi Meng, Yue-Li Zou, Hui Bu, Jun-Ying He \\ Department of Neurology, Second Hospital of Hebei Medical University, Shijiazhuang 050000, Hebei, China.
}

\section{A B S T R A C T}

Aim: Japanese encephalitis (JE) is caused by a mosquito-borne flavivirus and demonstrates high mortality and serious sequelae. Imaging and cytological examinations are important for the diagnosis of JE. We performed this study to analyze the imaging and cytological characteristics of JE. Methods: This study enrolled 92 JE patients with 108 cerebral spinal fluid (CSF) samples. Diagnosis was based on clinical features and positive immunoglobulin $\mathrm{M}$ antibodies against JE virus, which were measured using enzyme-linked immunosorbent assay. All patients received detailed neurological examinations, relevant cerebrospinal fluid tests, and brain neuroimaging (computed tomography, magnetic resonance imaging, or both). Results: Prominent involvement in the hippocampus was observed in 10 patients on neuroimaging, in addition to classic involvement in the thalamus and basal ganglia. Lumbar puncture pressure was normal in 61 CSF samples. White cell count increased in $81.19 \%$ of CSF samples, $67.65 \%$ and $83.33 \%$ of CSF samples demonstrated normal chloride and glucose concentrations, respectively, and $82.52 \%$ of CSF samples demonstrated $>0.4 \mathrm{~g} / \mathrm{L}$ protein content. JE patients demonstrate mixed-cell reaction on cerebrospinal fluid cytology in the early phase, which subsequently mainly develop as mainly lymphocyte reaction or typical lymphocyte reaction. Conclusion: JE imaging is characterized by bilateral thalamic involvement, and the basal ganglia and hippocampus are also commonly affected. The mixed-cell reaction in JE lasts longer than in general viral encephalitis. This may facilitate the differential diagnosis of JE.

Key words: Cerebrospinal fluid, cytology, Japanese encephalitis, neuroimaging

\section{INTRODUCTION}

Japanese encephalitis (JE), which is caused by infection with the JE virus (JEV), is one of the most important viral encephalitis in the world, especially in East and Southeast Asia. Approximately 35,000-50,000 people develop JE each year, demonstrating annual mortality of 10,000-15,000..$^{[1-3]}$ About one-third of patients die, and half of all survivors develop severe sequelae. ${ }^{[4-6]} \mathrm{JE}$ is characterized by high fever, conscious disturbance, seizures, focal neurological deficit, signs of meningeal irritation, etc. JE is regarded as a pediatric disease in endemic areas. The morbidity of JE has substantially

\begin{tabular}{|l|l|}
\hline \multicolumn{2}{|c|}{ Access this article online } \\
\hline Quick Response Code: & \\
\hline & Website: \\
\hline & Www.nnjournal.net \\
& DOI: \\
\hline
\end{tabular}

decreased due to the wide application of the JE vaccine, though outbreaks still occur in some districts and the number of adult infections is increasing. ${ }^{[7]}$ Bilateral thalamic lesions developed in endemic areas during susceptible seasons should be considered as encephalitis. Cytological analysis of the cerebrospinal fluid (CSF) may reflect the clinical course of JE. Here, we analyze the radiological and cytological features of JE.

\section{METHODS}

This study enrolled $92 \mathrm{JE}$ patients who were diagnosed and treated at the Second Hospital of Hebei Medical University between August 2013 and October 2013. Diagnosis was positive confirmation of immunoglobulin M (IgM) antibodies to JEV in sera using IgM antibody capture enzyme-linked immunosorbent assay by the center for disease control and prevention in Hebei province. All CSF samples were examined at Second Hospital of Hebei Medical University to determine the cellular, glucose, protein, chloride, and cytological characteristics. Ethics approval was given by the review board of the Second Hospital of Hebei Medical University. 
Cranial computed tomography (CT) scans were performed using a high-resolution CT scanner (GE OPTIA 660), and $10 \mathrm{~mm}$ axial sections were obtained parallel to the orbitomeatal plane. Magnetic resonance (Philips Achieva 3.0T) imaging (MRI) of the head was performed to obtain 3.0 T1-weighted spin-echo (repetition time [TR] $=2000$ $\mathrm{ms}$, echo time $[\mathrm{TE}]=20 \mathrm{~ms}$ ) and $\mathrm{T} 2$-weighted spin-echo images ( $\mathrm{TR}=1500 \mathrm{~ms}$; $\mathrm{TE}=80 \mathrm{~ms}$ ) in the axial, coronal, and sagittal planes. Section thickness was $6 \mathrm{~mm}$.

\section{RESULTS}

Ninety-two patients were included in this study, including 50 males and 42 females (male:female $=1.19: 1$ ), and the mean age was $42.32 \pm 15.47$ years. All patients (100\%) had fever, 53 patients (57.61\%) had headache, 74 patients (80.43\%) occurred consciousness disorder, 21 patients (22.83\%) demonstrated twitching, 34 patients $(36.96 \%)$ demonstrated dystonia, and 25 patients $(27.17 \%)$ demonstrated respiratory failure. Fifty patients (54.35\%) had sequelae at discharge following treatment.

Nineteen patients received CT examination of head between 2 and 25 days after onset, which were abnormal in 7 patients (36.84\%). Abnormalities included 1 patient (5.26\%) with thalamic hypodensity accompanied by basal ganglia and temporal lobe hypodensity, 4 patients (21.05\%) with basal ganglia hypodensity, 2 patients (10.53\%) with brain swelling, and 1 patient (5.26\%) with frontal lobe hypodensity.

Sixty-five patients received MRI of head within 2-32 days after onset. Lesions appeared hyperintense on fluid attenuated inversion recovery (FLAIR) and T2-weighted images, and isointense to slightly hypointense on T1-weighted images. MRI showed no lesions in 16 patients (24.62\%) within 2-22 days after onset. MRI findings of 30 patients with clear inflammatory focus included varying degrees of thalamic lesions except two patients. MRI lesions were also noted in the basal ganglia in 11 patients, mid-brain and hippocampus in 10 patients, pons in 1 patient, and cerebral cortex in 5 patients [Table 1].

Seventy-five patients underwent lumbar puncture, and 34 patients required puncture more than once. Symptom duration prior to lumbar puncture ranged between 3 and 33 days after onset. Hence, we identified 108 CSF samples ( 3 in the initial phase, 53 in the acute phase and 52 in convalescence). The mean lumbar puncture pressure was $173.41 \pm 77.87 \mathrm{mmH}_{2} \mathrm{O}$, and

\begin{tabular}{lcc}
\hline Table I: Different lesions identified using MRI & \\
\hline Features & $\mathbf{C T}(\boldsymbol{n}=\mathbf{2 0})$ & MRI $(\boldsymbol{n}=\mathbf{6 5})$ \\
\hline Abnormal & 7 & 30 \\
Thalamic & $1\left(1^{*}\right)$ & $28\left(20^{*}\right)$ \\
Basal ganglia & $4\left(3^{*}\right)$ & $11\left(8^{*}\right)$ \\
Hippocampus & 0 & $10\left(6^{*}\right)$ \\
Mid-brain & 0 & $10\left(4^{*}\right)$ \\
Pons & 0 & 1 \\
Cortex & 2 & $5\left(2^{*}\right)$ \\
Insult & 0 & 2 \\
White matter lesions & 1 & $6\left(2^{*}\right)$ \\
\hline${ }^{*}$ Number of bilateral lesions. CT: Computed tomography, MRI: Magnetic \\
resonance imaging
\end{tabular}

\begin{tabular}{lccc}
\hline Table 2: Different kinds of cells in different JE phases \\
\hline Cell type & $\begin{array}{c}\text { Initial } \\
\text { phase \% }\end{array}$ & $\begin{array}{c}\text { Acute } \\
\text { phase \% }\end{array}$ & $\begin{array}{c}\text { Convalescence } \\
\%\end{array}$ \\
\hline Lymphocytes & 35.67 & 71.06 & 89.68 \\
Monocytes & 19.33 & 12.27 & 4.7 \\
Activated monocytes & 15.67 & 9.51 & 2.82 \\
Neutrophils & 29.67 & 5.84 & 2.49 \\
Plasma cells & 0.67 & 1.14 & 0.16 \\
Lymphoid cells & 0 & 0 & 0.12 \\
Eosinophils & 0 & 0.04 & 0.04 \\
Basophils & 0 & 0.08 & 0 \\
\hline
\end{tabular}

JE: Japanese encephalitis

27 samples (25.71\%) demonstrated high lumbar puncture pressure and 61 samples (58.10\%) demonstrated normal lumbar puncture pressure, 3 samples weren't tested for pressure. The mean number of white cells in CSF was $(42.50 \pm 71.31) \times 10^{6} / \mathrm{L}$, and $81.19 \%$ of samples demonstrated an increase in their white cell count. Mean chloride, glucose, and protein values were $122.26 \pm 9.02 \mathrm{mmol} / \mathrm{L}, 3.77 \pm 1.79 \mathrm{mmol} / \mathrm{L}$, $0.64 \pm 0.28 \mathrm{~g} / \mathrm{L}$, respectively. In total, $67.65 \%$ and $83.33 \%$ of samples demonstrated normal chloride and glucose samples (normal chloride content: 120-130 mmol/L; normal glucose content: $2.5-4.4 \mathrm{mmol} / \mathrm{L}$ ), while $82.52 \%$ of samples demonstrated $>0.4 \mathrm{~g} / \mathrm{L}$ protein content. The cytological examination of CSF confirmed mixed-cell reaction in 11 (10.19\%) samples, an increase in activated monocytes in $40(37.04 \%)$ samples (lymphocytes were the main cells in 35 (32.41\%) samples), mainly lymphocyte reaction in 41 (37.96\%) samples (neutrophil were found in 15), typical lymphocyte reaction in $12(11.11 \%)$ samples, and $<100$ cells in $4(3.7 \%)$ samples. We also analyzed the proportion of various cells in different JE phases [Table 2].

\section{DISCUSSION}

The imaging findings obtained using CT and MRI showed the pathological changes that occur in JE patients. JE patients typically demonstrate hypodense lesions in the thalamus and basal ganglia on CT. MRI is more sensitive than CT for revealing abnormalities, ${ }^{[8]}$ 
and MRI demonstrates a higher diagnostic value than CT, especially when assessing site, range, quantity, and extent of infection. Thalamic changes, especially bilateral involvement, could be used to help diagnosis JE in endemic areas.

Lesions can also be observed in the substantia nigra, brain stem, cerebellum, cerebral cortex, and white matter. ${ }^{[8-10]}$ One study reported changes in the thalamus (94\%), basal ganglia (35.5\%), midbrain (58\%), cerebellum (25.8\%), pons (19\%), and cerebral cortex (19\%) on MRI. ${ }^{[8]}$ MRI lesions are generally hypointense on $\mathrm{T} 1$ and hyperintense on T2 and FLAIR images. FLAIR is the most useful sequence for detecting lesions and defining the extent of supratentorial lesions. On FLAIR images, cerebral lesions can be better observed, but T2-weighted imaging is better for evaluating the midbrain and brain stem. ${ }^{[11]}$ Thalamic lesions may demonstrate mixed intensity on $\mathrm{T} 1$ and $\mathrm{T} 2$ imaging in the subacute phase, which may be suggestive of hemorrhagic changes. ${ }^{[12]}$ The involvement of temporal lobe has also been observed in some studies, ${ }^{[8,13,14]}$ but all reported patients also demonstrated involvement of the thalamus and substantia nigra. ${ }^{[14]}$ Temporal lobe involvement is fairly characteristic and mostly involves the hippocampus, usually sparing the rest of the temporal lobe. Because JEV takes a hematogenous route during the invasion and infects the blood supply in parts of the thalamus, cerebral peduncles, hippocampus, and uncus, ${ }^{[14]}$ this may explain concurrent involvement in the medial temporal lobe along with the thalami and substantia nigra. The hippocampal involvement was most commonly in the tail and body, occasionally in the head and amygdala. ${ }^{[14,15]}$ The presence of typical JE lesions in the thalami, substantia nigra, and basal ganglia, along with temporal lobe involvement, may help differentiate JE from herpes simplex virus encephalitis (HSE). Sawlani ${ }^{[11]}$ has reported that MRI techniques such as FLAIR and diffusion weighted imaging (DWI) can be used to evaluate HSE and JE. DWI and apparent diffusion coefficients mapping (ADC) can differentiate cytotoxic edema from vasogenic edema. ${ }^{[16]}$ Significantly restricted diffusion and low average ADC values have been observed in the acute phase lesions in HSE patients, whereas JE lesions do not show restricted diffusion or significantly low ADC values in the acute phase (whereas chronic phase lesions show restricted diffusion and high ADC values). ${ }^{[11]}$ However, Prakash et al. ${ }^{[17]}$ have reported restricted diffusion and low ADC values in the acute phase JE. Coinfection of neurocysticercosis (NCC) and JE has also been observed by MRI and CT, which may result in poor clinical outcomes. ${ }^{[13,18]}$ A significantly higher proportion of abnormal CT scans and abnormal MRI has been reported when evaluating NCC/JE-coinfected lesions. ${ }^{[18]} \mathrm{JE}$ in association with cerebral venous sinus thrombosis has also been reported with the help of MRI and MR venography. ${ }^{[19]}$

In this study, 65 patients took MRI examination in 92 patients. Thirty patients (46.15\%) presented with inflammatory lesions between 2 and 32 days after onset, 3 patients $(10.00 \%)$ presented in the initial phase, 19 patients $(63.63 \%)$ in the acute phase, and 8 patients $(26.67 \%)$ in convalescence. Handique ${ }^{[15]}$ has reported $90.00 \%$ MRI sensitivity during the 1st week of JE infection. However, in this study, 13 of 30 (43.33\%) patients presented with lesions on MRI during the 1st week of JE infection. Our patients were first treated at other hospitals, and some patients were too ill for early examination, so their MRI examinations were not timely, which may explain the low sensitivity in this study.

However, this study does show changes in the thalamus (93.33\%), basal ganglia (36.67\%), hippocampus (33.33\%), mid-brain (33.33\%), pons (3.33\%), and cerebral cortex (16.67\%) on MRI [Figure 1]. Except for one patient, all patients demonstrated hippocampal involvement on MRI that was accompanied by thalami involvement. The gray matter areas of the brain, including the thalamus and hippocampus, were primarily affected by JEV on autopsy. ${ }^{[20]}$ These areas are associated with increases in activated and phagocytic microglia. ${ }^{[21]}$ Srivastava et al. ${ }^{[22]}$ have reported that JEV RNA load in different brain regions of rat with higher affinity of JEV to thalamus and mid brain compared to other regions. These may explain the commonly affected areas observed on imaging. Three patients were examined using both CT and MRI, but only MRI revealed lesions. One MRI reexamination reported lesion reduction following treatment, while another reported enlarged lesions. That may be the first study that used early MRI examination and did not observe the peak of brain injury. Three patients showed inflammatory lesions in combination with ischemic infarction on MRI. All of these patients demonstrated risk factors that are also shared with severe encephalitis, such as hypertension and age. Basumatary et al. ${ }^{[4]}$ have reported that changes on MRI or CT in combination with thalamic involvement are significantly related with dystonia. However, other clinical symptoms, such as behavioral abnormalities, seizure, coma level, and death, do not demonstrate a significant correlation with radiological abnormalities. 

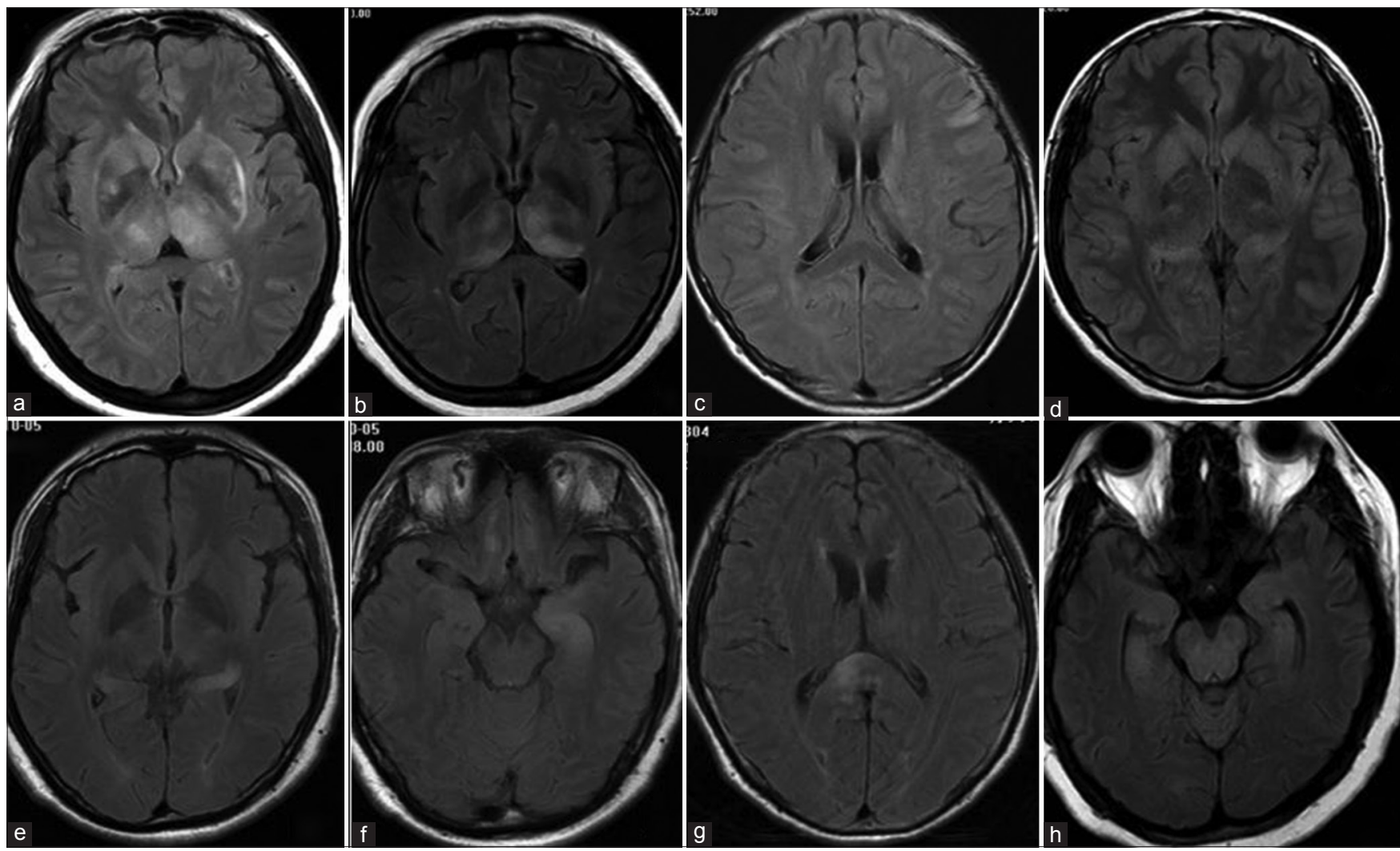

Figure 1: Axial fluid attenuated inversion recovery images of involvement in different areas: (a) Involvement in the bilateral thalamus and basal ganglia; (b) Involvement in the bilateral thalamus; (c) Involvement in the left prefrontal cortex; (d) Involvement in the bilateral frontal lobe, temporal lobe, insula cortex, thalamus, and basal ganglia; (e) Involvement in the bilateral hippocampal tail; (f) Involvement in the bilateral hippocampal head and body and uncus; ( $g$ ) Involvement in the corpus callosum; (h) Involvement in the bilateral hippocampal head and body and midbrain

We did not observe NCC and JE coinfection in this study, but there was a forty-year-old woman who got JE associated with cerebral venous sinus thrombosis. The MRI displayed the swelling of the left frontal, parietal lobes and the right occipital with hypointense on T1 and hyperintense on T2 and FLAIR images. The superior sagittal sinus, left sigmoid sinus and transverse sinus filling defect were observed, indicating venous thrombosis. MRV scan further demonstrated thrombosis in the superior sagittal sinus, left sigmoid sinus and transverse sinus, confirming the diagnosis of CVST. Twenty-six patients presented with dystonia, including 16 patients (61.54\%) who demonstrated MRI changes with thalamic involvement. Another $16 \mathrm{JE}$ patients did not show lesions on MRI within 6-22 days after onset. This may indicate that thalamic abnormalities suggest JE, but absence does not exclude it. In additional, 11 of 30 patients with MRI abnormalities also received DWI examinations, and 9 of these patients demonstrated lesions with abnormal signals on both T2-weighted imaging and DWI within 5-12 days (mean $=5.9$ days) after onset. Due to the decrease in the vasculitis component and perivascular cuffing, the proportion of diffusion restriction decreased and ADC began to rise in the late-acute and early-subacute phases. ${ }^{[17]} \mathrm{In}$ this phase, cytotoxic edema is accompanied by the vasogenic collection of fluid that allows the lesion to become visible on both T2W and DWI.

CSF parameters may change in JE patients when the brain tissues or meninges are involved. To summarize, lumbar puncture pressure was normal or slightly increased in most patients. White blood cell count and protein content increased slightly, similar to related reports, ${ }^{[23,24]}$ while the glucose and chloride content were normal in most JE patients. This increase in protein reflects the increase of endothelial cell permeability, which indicates that the blood-brain barrier is damaged in JE patients. The cytological features of CSF are associated with the clinical course of JE, and performance varies in different phases of disease. We analyzed $108 \mathrm{CSF}$ samples and observed the mixed-cell reaction in the early phase of JE. Neutrophils were the major inflammatory cells (mean $=35.67 \%$ ), though neutrophil predominance is not uncommon in other viral central nervous system infections. ${ }^{[25]}$ We also identified activated monocytes and plasma cells during the disease course. In the acute phase of JE, the proportion 


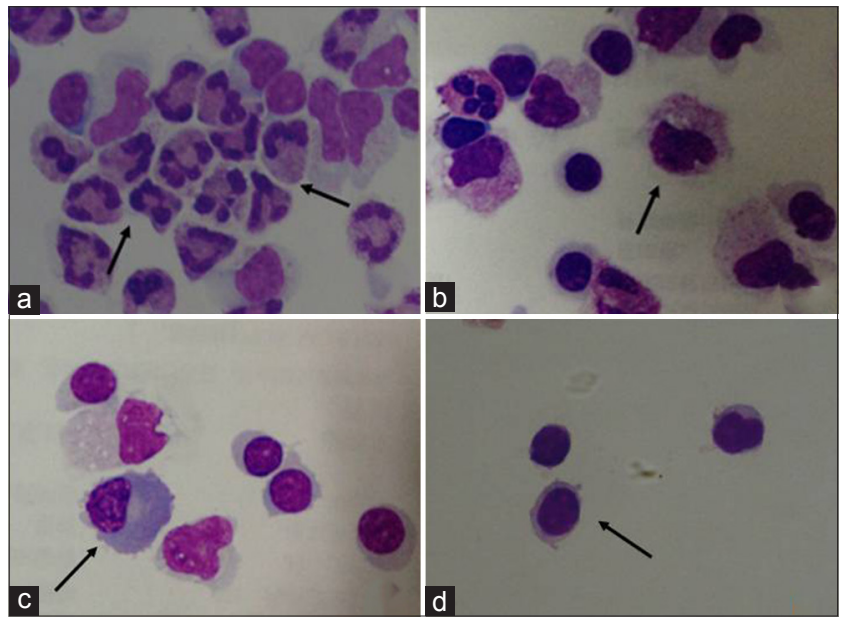

Figure 2: May-Grünwald-Giemsa staining results $(\times 1000)$ of the cerebral spinal fluid in Japanese encephalitis patient at different phases: (a) Initial phase - Neutrophils (black arrows) are the main inflammatory cells in the background; (b) Acute phase - A decrease in the number of neutrophils and an increase in the number of lymphocytes were noted. Furthermore, activated monocytes (black arrows) are apparent; (c) Acute phase - Plasmocytes developed in the acute phase (black arrows); (d) Convalescence - Cytology mainly shows the lymphocyte reaction (black arrows)

of neutrophils dramatically declined to $5.82 \%$, while lymphocytes significantly increased. Monocytes and activated monocytes gradually decreased, but plasma cells peaked. At convalescence, mainly lymphocyte reaction or typical lymphocyte reaction were observed on CSF cytological examination. Neutrophils, monocytes, activated monocytes, and plasma cells all rapidly decreased or even disappeared [Figure 2]. In addition to typical cytological characteristics of viral encephalitis, CSF cytological examination of JE patients also showed that the mixed-cell reaction existed for long periods of time, and we observed neutrophils existed for longer than one week. The rates of neutrophils decrease and lymphocyte increase are fast during treatment, and this may facilitate the differential diagnosis of JE. Misra and Kalita ${ }^{[26]}$ have reported that the 3-month clinical outcomes were not significantly related to CSF abnormalities. Further studies are required.

Based on the results of routine biochemical examinations of CSF, white blood cell and protein content slightly increased while glucose and chloride content remained normal in most JE patients. The mixed-cell reaction was noted in the early phase of JE and existed longer than in general viral encephalitis. At convalescence, cytological examinations of CSF demonstrated mainly lymphocyte reaction or typical lymphocyte reaction. The mixed-cell reaction in JE lasts longer than in general viral encephalitis. JE imaging is characterized by bilateral thalamic involvement, and involvement of the basal ganglia and hippocampus are also common.
In endemic areas and epidemic season, the patients considered as CNS infection with bilateral thalamic involvement should be highly suspected as JE.

\section{REFERENCES}

1. Solomon T. Control of Japanese encephalitis - within our grasp? $N$ Engl J Med 2006;355:869-71.

2. Erlanger TE, Weiss S, Keiser J, Utzinger J, Wiedenmayer K. Past, present, and future of Japanese encephalitis. Emerg Infect Dis 2009; 15:1-7.

3. Gao X, Nasci R, Liang G. The neglected arboviral infections in mainland China. PLoS Negl Trop Dis 2010;4:e624.

4. Basumatary LJ, Raja D, Bhuyan D, Das M, Goswami M, Kayal AK. Clinical and radiological spectrum of Japanese encephalitis. JNeurol Sci 2013;325:15-21.

5. Ghosh D, Basu A. Japanese encephalitis-a pathological and clinical perspective. PLoS Negl Trop Dis 2009;3:e437.

6. Solomon T, Dung NM, Kneen R, Gainsborough M, Vaughn DW, Khanh VT. Japanese encephalitis. J Neurol Neurosurg Psychiatry 2000;68:405-15.

7. Zheng Y, Li M, Wang H, Liang G. Japanese encephalitis and Japanese encephalitis virus in mainland China. Rev Med Virol 2012;22:301-22.

8. Kalita J, Misra UK. Comparison of CT scan and MRI findings in the diagnosis of Japanese encephalitis. J Neurol Sci 2000;174:3-8.

9. Misra UK, Kalita J, Phadke RV, Wadwekar V, Boruah DK, Srivastava A, Maurya PK, Bhattacharyya A. Usefulness of various MRI sequences in the diagnosis of viral encephalitis. Acta Trop 2010;116:206-11.

10. Dung NM, Turtle L, Chong WK, Mai NT, Thao TT, Thuy TT, Kneen R, Phu NH, Wills B, Farrar J, Das K, Solomon T. An evaluation of the usefulness of neuroimaging for the diagnosis of Japanese encephalitis. J Neurol 2009;256:2052-60.

11. Sawlani V. Diffusion-weighted imaging and apparent diffusion coefficient evaluation of herpes simplex encephalitis and Japanese encephalitis. J Neurol Sci 2009;287:221-6.

12. Misra UK, Kalita J. Overview: Japanese encephalitis. Prog Neurobiol 2010;91:108-20.

13. Singh P, Kalra N, Ratho RK, Shankar S, Khandelwal N, Suri S. Coexistent neurocysticercosis and Japanese B encephalitis: MR imaging correlation. AJNR Am J Neuroradiol 2001;22:1131-6.

14. Handique SK, Das RR, Barman K, Medhi N, Saharia B, Saikia P, Ahmed SA. Temporal lobe involvement in Japanese encephalitis: problems in differential diagnosis. AJNR Am J Neuroradiol 2006;27:1027-31.

15. Handique SK. Viral infections of the central nervous system. Neuroimaging Clin N Am 2011;21:777-94, vii.

16. Agid R, Ducreux D, Halliday WC, Kucharczyk W, terBrugge KG, Mikulis DJ. MR diffusion-weighted imaging in a case of West Nile virus encephalitis. Neurology 2003;61:1821-3.

17. Prakash M, Kumar S, Gupta RK. Diffusion-weighted MR imaging in Japanese encephalitis. J Comput Assist Tomogr 2004;28:756-61.

18. Handique SK, Das RR, Saharia B, Das P, Buragohain R, Saikia P. Coinfection of Japanese encephalitis with neurocysticercosis: an imaging study. AJNR Am J Neuroradiol 2008;29:170-5.

19. Jia M, Xiong N, Huang J, Wang Y, Zhang X, Zhang Z, Cao X, Lin Z, Wang T. Japanese encephalitis accompanied by cerebral venous sinus thrombosis: a case report. BMC Neurol 2012;12:43.

20. Sips GJ, Wilschut J, Smit JM. Neuroinvasive flavivirus infections. Rev Med Virol 2012;22:69-87.

21. Ghoshal A, Das S, Ghosh S, Mishra MK, Sharma V, Koli P, Sen E, Basu A. Proinflammatory mediators released by activated microglia induces neuronal death in Japanese encephalitis. Glia 2007;55:483-96. 
22. Srivastava R, Kalita J, Khan MY, Gore MM, Bondre VP, Misra UK. Temporal changes of Japanese encephalitits virus in different brain regions of rat. Indian $J$ Med Res 2013;138:219-23.

23. Ayukawa R, Fujimoto $H$, Ayabe M, Shoji H, Matsui R, Iwata Y, Fukuda H, Ochi K, Noda K, Ono Y, Sakai K, Takehisa Y, Yasui K. An unexpected outbreak of Japanese encephalitis in the Chugoku district of Japan, 2002. Jpn J Infect Dis 2004;57:63-6.

24. Takamatsu Y, Uchida L, Nga PT, Okamoto K, Nabeshima T, Thao DT, Hai do T, Tuyet NT, Duc HM, Luat le X, Hasebe F, Morita K. An approach for differentiating echovirus 30 and Japanese encephalitis virus infections in acute meningitis/encephalitis: a retrospective study of 103 cases in Vietnam. Virol J 2013;10:280.

25. Tyler KL, Pape J, Goody RJ, Corkill M, Kleinschmidt-DeMasters BK.
CSF findings in 250 patients with serologically confirmed West Nile virus meningitis and encephalitis. Neurology 2006;66:361-5.

26. Misra UK, Kalita J. Prognosis of Japanese encephalitis patients with dystonia compared to those with parkinsonian features only. Postgrad Med J 2002;78:238-41.

Cite this article as: Meng Q, Zou YL, Bu H, He JY. Imaging and cytological analysis of 92 patients with Japanese encephalitis. Neuroimmunol Neuroinflammation 2014;1(1):29-34

Source of Support: The center for disease control and prevention in Hebei province. Conflict of Interest: No.

Received: 15-04-2014; Accepted: 29-05-2014 